\title{
FINITE LOOP SPACES WITH MAXIMAL TORI HAVE FINITE WEYL GROUPS
}

\author{
LARRY SMITH
}

(Communicated by Frederick R. Cohen)

\begin{abstract}
A finite loop space $X$ is said to have a maximal torus if there is a map $f: B T \rightarrow B X$ where $T$ is a torus such that $\operatorname{rank}(T)=\operatorname{rank}(X)$ and the homotopy fibre of $f$ has the homotopy type of a finite complex.

The Weyl group $W_{f}$ of $f$ is the set of homotopy classes $w: B T \rightarrow B T$ such that

$$
\begin{gathered}
B T \stackrel{w}{\rightarrow} B T \\
B X{ }^{\swarrow} f
\end{gathered}
$$
\end{abstract}

homotopy commutes. In this note we prove that $W_{f}$ is always finite.

By a finite loop space we understand a topological space $X$ such that:

- $X$ has the homotopy type of a finite complex,

- $X$ has the homotopy type of a topological group.

$N . B$. It is not assumed both structures may be realized on the same space simultaneously.

A compact Lie group is a finite loop space, but there are well-known examples (see, e.g., the book of Richard Kane [2] and the references there) of finite loop spaces which are not Lie groups. An important idea, introduced by Rector [7] (see also [6, I, II, III; 5]) for the study of finite loop spaces is that of a maximal torus.

Definition. If $X$ is a finite loop space, a maximal torus for $X$ is a map $f: B T \rightarrow B X$ where $B(-)$ is the classifying space functor and $T$ is a torus such that

- $\operatorname{rank}(T)=\operatorname{rank}(X)$ (see next page for definition of rank),

- the homotopy fibre $F$ of $f$ has the homotopy type of a finite complex.

A finite loop space need not have a maximal torus as Rector showed [7], and if $X$ is a fake Lie group, then it has a maximal torus if and only if it is a Lie group [6, I-III; 5]. For a finite loop space with maximal torus, Rector adapted a further concept from Lie theory, namely, the Weyl group.

Received by the editors August 23, 1991.

1991 Mathematics Subject Classification. Primary 55F35. 
Definition. If $X$ is a finite loop space with a maximal torus $f: B T \rightarrow B X$, then the Weyl group of $f, W_{f}$, is the group of homotopy classes of maps $w: B T \rightarrow B T$ such that

$$
\begin{gathered}
B T \stackrel{w}{\rightarrow} B T \\
f \searrow{ }_{B X}
\end{gathered}
$$

homotopy commutes.

Notice that $[B T, B T] \simeq \mathrm{GL}(n ; \mathbb{Z})$, where $n=\operatorname{rank}(T)$, so $W_{f} \leq \mathrm{GL}(n ; \mathbb{Z})$. If $G$ is a compact connected Lie group and $X$ a fake Lie group (e.g., $X=G$ ) of type $G$ (see [6, I] for definitions) with a maximal torus $f: B T \rightarrow B X$ (e.g., $f=B_{\rho}$ where $\rho: T \hookrightarrow G$ is the inclusion of a maximal torus), then $W_{f} \simeq W_{G}$. However, for a general finite loop space there seems no reference for the fact that $W_{f}$ is a finite group. ${ }^{\dagger}$ We rectify this in the following:

Theorem. Let $X$ be a connected finite loop space and suppose $X$ has a maximal torus $f: B T \rightarrow B X$. Then the Weyl group $W_{f}$ is finite, and $\left|W_{f}\right|$ is a divisor of $d=d_{1} \cdots d_{n}$ where $\left(2 d_{1}-1, \ldots, 2 d_{n}-1\right)$ is the type of $X$.

The type of a finite loop space $X$ (or $H$-space) is defined following Hopf (see, e.g., [3]) who showed

$$
H^{*}(X ; \mathbb{Q})=H^{*}\left(S^{2 d_{1}-1} \times \cdots \times S^{2 d_{n}-1} ; \mathbb{Q}\right)
$$

where the integers $2 d_{1}-1, \ldots, 2 d_{n}-1$ are called the type of $X$ and $n$ its rank. If $X$ is a Lie group, then the rank as just defined coincides with its rank as a Lie group.

Proof. Let $X$ have rank $n$ and type $\left(2 d_{1}-1, \ldots, 2 d_{n}-1\right)$. By the LeraySamelson theorem [3] it follows that for sufficiently large primes $p$ or $p=0$

$$
H^{*}\left(X ; \mathbb{F}_{p}\right) \simeq E\left(u_{1}, \ldots, u_{n}\right)
$$

where $\left(\mathbb{F}_{p}:=\mathbb{Z} / p, \mathbb{F}_{0}=\mathbb{Q}\right)$

$$
\operatorname{deg} u_{i}=2 d_{i}-1, \quad i=1, \ldots, n .
$$

From the Milnor-Moore spectral sequence [4] it then follows

$$
H^{*}\left(B X, \mathbb{F}_{p}\right) \simeq \mathbb{F}_{p}\left[\rho_{1}, \ldots, \rho_{n}\right]
$$

where

$$
\operatorname{deg} \rho_{i}=2 d_{i}, \quad i=1, \ldots, n .
$$

Let $F$ denote the fibre of the maximal torus $f: B T \rightarrow B X$. Consider the Serre spectral sequence $\left\{E_{r}, d_{r}\right\}$

$$
\begin{aligned}
& E_{r} \Rightarrow H^{*}\left(B T ; \mathbb{F}_{p}\right), \\
& E_{2}=H^{*}\left(B X ; \mathbb{F}_{p}\right) \otimes H^{*}\left(F ; \mathbb{F}_{p}\right) .
\end{aligned}
$$

Since $F$ is finite, $E_{2}^{* *}$ is a finitely generated $H^{*}\left(B X ; \mathbb{F}_{p}\right)$ module. If $p=0$, or $p$ is sufficiently large, $H^{*}\left(B X ; \mathbb{F}_{p}\right)$ is a noetherian ring, and hence an easy argument shows that $H^{*}\left(B T ; F_{p}\right)$ is a finitely generated $H^{*}\left(B X ; \mathbb{F}_{p}\right)$ module. Recall

$$
H^{*}\left(B T ; \mathbb{F}_{p}\right) \simeq \mathbb{F}_{p}\left[t_{1}, \ldots, t_{n}\right]
$$

' In [8] Rector and Stasheff state that this is the case but give no proof. 
where

Therefore,

$$
\operatorname{deg} t_{i}=2, \quad i=1, \ldots, n \text {. }
$$

$$
f^{*}: H^{*}\left(B X ; \mathbb{F}_{p}\right) \rightarrow H^{*}\left(B T ; \mathbb{F}_{p}\right)
$$

must be monic for $H^{*}\left(B T ; \mathbb{F}_{p}\right)$ to be finitely generated over $H^{*}\left(B X ; \mathbb{F}_{p}\right)$, and furthermore by Maccauley's theorem [9] $H^{*}\left(B T ; \mathbb{F}_{p}\right)$ is a free $H^{*}\left(B X ; \mathbb{F}_{p}\right)$ module.

From this point on assume that $p$ is a prime that is sufficiently large and $p \nmid d=d_{1} \cdots d_{n}$. Then according to Adams-Wilkerson [1] there exists an essentially unique embedding

$$
\varphi: H^{*}\left(B X ; \mathbb{F}_{p}\right) \hookrightarrow H^{*}\left(B T ; \mathbb{F}_{p}\right)
$$

and a finite group $W(p) \leq \mathrm{GL}\left(n ; \mathbb{F}_{p}\right)$ with

$$
\varphi\left(H^{*}\left(B X ; \mathbb{F}_{p}\right)\right)=H^{*}\left(B T ; \mathbb{F}_{p}\right)^{W(p)}
$$

and

$$
|W(p)|=d .
$$

The uniqueness of $\varphi$ allows us to suppose that $\varphi=f^{*}$. By the very definition of $W_{f}$ we have

$$
H^{*}\left(B T ; \mathbb{F}_{p}\right)^{W(p)} \leq H^{*}\left(B T ; \mathbb{F}_{p}\right)^{W_{f}} .
$$

Let $F F(-)$ denote the field of fractions functor, and set $H^{*}\left(B T ; \mathbb{F}_{p}\right)=B$. Then

$$
F F(B)^{W(p)}=F F\left(B^{W(p)}\right) \leq F F\left(B^{W_{f}}\right) \leq F F(B)^{W_{f}}
$$

where the first equality results from [6, I.3.2]. By Galois theory it follows that the image of $W_{f}$ under the reduction homomorphism

$$
\rho: \operatorname{GL}(n ; \mathbb{Z}) \rightarrow \mathrm{GL}\left(n ; \mathbb{F}_{p}\right)
$$

is contained in $W(p)$.

Suppose that $\left|W_{f}\right|>d$. Let $1=w_{0}, w_{1}, \ldots, w_{d} \in W_{f}$ be $d+1$ distinct elements. Choose $p$ even larger if necessary so that

$$
p \nmid w_{r}(i, j)-w_{s}(i, j):\left\{\begin{array}{l}
0 \leq r, s \leq d, \\
1 \leq i, j \leq n .
\end{array}\right.
$$

Then the $\bmod p$ reduction of these elements would have to be distinct, contrary to the fact

$$
\rho\left(W_{f}\right) \leq W(p), \quad|W(p)|=d .
$$

Therefore, $W_{f}$ is finite, and $p$ is monic if $p$ is large enough.

Remark. The referee has suggested an alternate proof. In outline the idea is as follows. Work over the rationals $\mathbb{Q}$ instead of the finite field $\mathbb{Z} / p$. As above $A:=H^{*}(B T ; \mathbb{Q})$ is a finitely generated module over $B:=H^{*}(B X ; \mathbb{Q})$. (One must argue that $H^{*}(B X ; \mathbb{Q}) \rightarrow H^{*}(B T ; \mathbb{Q})$ is monic and:) Therefore $A \supseteq B$ is an integral ring extension. The canonical map $W \rightarrow \operatorname{Aut}(F F(A))$ of the Weyl group $W$ into the automorphism group of the field of fractions of $A$ is an injection. $F F(A) \supseteq F F(A)^{W} \supseteq F F(B)$ is a sequence of field extensions, where the first is a Galois extension. An argument using Poincare series (e.g., T. Springer, Invariant theory, Lecture Notes in Math., vol. 585, 
$\S 2.5 .6$, Springer, New York) shows that the degree $[F F(A): F F(B)]$ of the extension $F F(A) \supseteq F F(B)$ is given by $\prod d_{i}$. Hence, the order of $W$ divides $\prod d_{i}$, and in particular, the order of $W$ is finite.

N.B. The paper of Rector-Stasheff [8] was published in 1974 and the book of Springer was first published in 1977.

The preceding theorem answers yet one more question about the Weyl group of a maximal torus for a finite loop space but leaves open many more. In particular:

- Is the Weyl group of a finite loop space with maximal torus nontrivial?

- Is $H^{*}\left(B X ; \mathbb{F}_{p}\right)$ the ring of invariants of $W_{f}$ acting on $H^{*}\left(B T ; \mathbb{F}_{p}\right)$ ?

Affirmative answers would classify the possible Weyl groups as the groups generated by reflections and answer in the affirmative the question of whether the type of a finite loop space with maximal torus must coincide with that of a Lie group.

\section{ACKNOWLEDGMENT}

I would like to thank the members of the Yale Mathematics Department for their hospitality during my stay and to the Ministieurm für Wissenschaft und Kultur in Hannover for relieving me of teaching responsibilities during the 1990 winter semester.

\section{REFERENCES}

1. J. F. Adams and C. W. Wilkerson, Polynomial algebras over the Steenrod algebra, Ann. of Math. (2) 110 (1980), 96-143.

2. R. M. Kane, Homology of Hopf spaces, North-Holland, Amsterdam, 1988.

3. J. Milnor and J. C. Moore, On the structure of Hopf Algebras, Ann. of Math. (2) 81 (1965). 211-264.

4. J. C. Moore, Algèbre homologique et homologie des éspaces classifiant, Seminar Cartan 1959/60 Expose 7.

5. D. Notbohm, Maps between classifying spaces and applications, Math. Göttingensis, vol. 20, Springer, Berlin and New York, 1991.

6. D. Notbohm and L. Smith, Fake Lie groups and maximal tori. I, II, III, Math. Ann. 288 (1990), 637-661; Math. Ann. 288 (1990), 663-673; Math. Ann. 290 (1991), 629-642.

7. D. Rector, Subgroups of finite dimensional topological groups, J. Pure Appl. Algebra 1 (1971), 253-273.

8. D. Rector and J. D. Stasheff, Lie groups from a homotopy point of view, Symposium on Algebraic Topology (Seattle), Lecture Notes in Math., vol. 249, Springer, Berlin, Heidelberg. and New York, 1971, pp. 99-105.

9. O. Zariski and P. Samuel, Commutative algebra, Vol. II (Appendix 7), van Nostrand. Princeton, NJ, 1958.

Department of Mathematics, Yale University, New Haven, Connecticut 06520

Mathematisches Institut, Bunsenstrasse 3/5, D34 Göttingen, Germany 